\title{
Application of Domestic Virtualization Software in Electric Information System
}

\author{
Hu Cong, Tang Yixuan, Guo Yang \\ Anhui Electric Power Corporation, Information \& Telecommunication Branch,Hefei,China \\ e-mail: huc0019@163.com
}

Keywords: cloud computing; virtualization; resource pool

\begin{abstract}
Aiming at the increasingly expanding business, large server maintenance amount and high maintenance cost, the Anhui Electric Power Corporation decided to carry out information system hardware resource pool construction based on advanced information technologies such as cloud computing and virtualization so as to improve hardware resource use efficiency, save machine room space and reduce operation cost etc. Anhui Electric Power Corporation verified the cross-memory and migration function of virtual machines with domestic virtualization software in the resource pool construction and application process, which improves the working efficiency effectively.
\end{abstract}

\section{Introduction}

At present, the enterprise hardware resource pool of most company adopts foreign commercial software VMware virtual products[1,2], which is characterized by high overall cost and low controllability and security. Based on this, Anhui Electric Power Corporation conduct the domestic domestic virtual software product construction and application aiming to achieve the substitution of foreign commercial virtualization software involved in the "resource pool" project construction, reduce the construction cost and improve the security as well as controllability.

\section{BACKGROUND}

At present, Anhui Electric Power Corporation adopts foreign commercial software VMware virtualization product concerning the software and hardware resource pool projects, which is characterized by relatively high overall cost as well as low controllability and security. Therefore, Anhui Electric Power Corporation carried out domestic virtual software product construction and application so as to achieve the substitution of foreign commercial virtualization software involved in the "resource pool" project construction, reduce the construction cost and improve the security as well as controllability. The main technologies are as follows:

(1) Two-engine technology

The two-engine technology supports KVM and XEN. That is, the two-engine technology supports KVM and XEN in the same cluster. Such fine grit can satisfy more complex demands, which is the first case in China. At present, the China only supports KVM or XEN single engine software.

(2) Energy saving and emission reduction technology

The energy saving and emission reduction technology was developed. The software was further used to construct intelligent monitoring module and conduct in-depth interaction with PC group. According to practical resource consumption, this technology is characterized by automatic, intelligent dispatching hardware and dynamic, migrate virtual machine.

(3) Support graphics processor

The graphics processor adapter was domestic developed. The local graphics were adopted to render direct-connected technology. The strong computing power of graphics processor was used to provide local graphics rendering service for virtual machines and solve the urgent demands concerning the high graphics card requirements.

(4) No hard disk leads rapid deploy technology 
The memdisk and squashfs technologies were used to store RO data of Hypervisor operating system on $C D$ or $U$ disk and then put the written data in the internal storage. Further, the aufs technology was adopted to splice the internal storage or CD or U disk into a virtual disk, which achieved Hypervisor operation system led by no hard disk and rapid (no hard disk) deployment physical server virtualization environment.

\section{TECHNICAL SCHEME}

According to the business demand as well as the investigation and deep analysis on the current situation of cloud computing, this paper decided to integrate grid model and virtualization technology based on OSGi[3,4] and adopted J2EE technology to conduct system development. The OSGi technology refers to Java-oriented dynamic model systems. OSGi service platform provided service to Java. Those services allow Java to become the preferred environment for software integration and development. OSGi technology provides and allows the application software to use refined, reusable and synergistic standard primitives constructed by the components.

OSGi service platform provides structure changes dynamically on various network equipments without restart. In order to minimize coupling degree and promote manageable coupling degree, OSGi technology provides a service-oriented framework that can allow those components to discover the others dynamically.

The cloud computing resource management interface based on REST open standard is a lightweight solution, which is characterized by better long-term compatibility and smaller dependency. Meanwhile, it can use cache to improve response speed. Further, it provides a method to interact with cloud computing resources. Many functions of cloud computing resource management can be implemented through this simple interface so as to manage a large number of resources in the cloud computing environment.

The cloud computing platform applied network to integrate various resources including computing resource, storage resource, network resource and software resources into a logic unit. The model abstraction based on grid was used to conduct dispatching management and provide users with integrated computing resource, information and application service. The basic information as well as real-time index data of all resources is stored in the grid completely. According to the data access frequency, the data is stored via memory mode and disk mode. The real-time ultra-high frequency data are stored via all-in-memory mode. The query speed of grid is ten times faster than the normal database. Meanwhile, the resource management service can manage multiple database centers and conduct centralized dispatching and management on various data centers.

Libvirt[5] is an open standard APT based on abstract conception, which supports various virtual-machine programs including KVM, Xen, VMWare, HyperV as well as other virtualization products. Meanwhile, it provides API for common functions realized by virtualization programs. In order to support the expandability of various virtual-machine monitors, Libvirt realization refers to a framework based on drive programs, which allows a common API to provide service for a large number of different types of virtual machine programs via general methods. The research on Libvirt open standard API achieves the dispatching and management on various types of virtualization platforms. 


\section{APPLICATION FRAMEWORK}

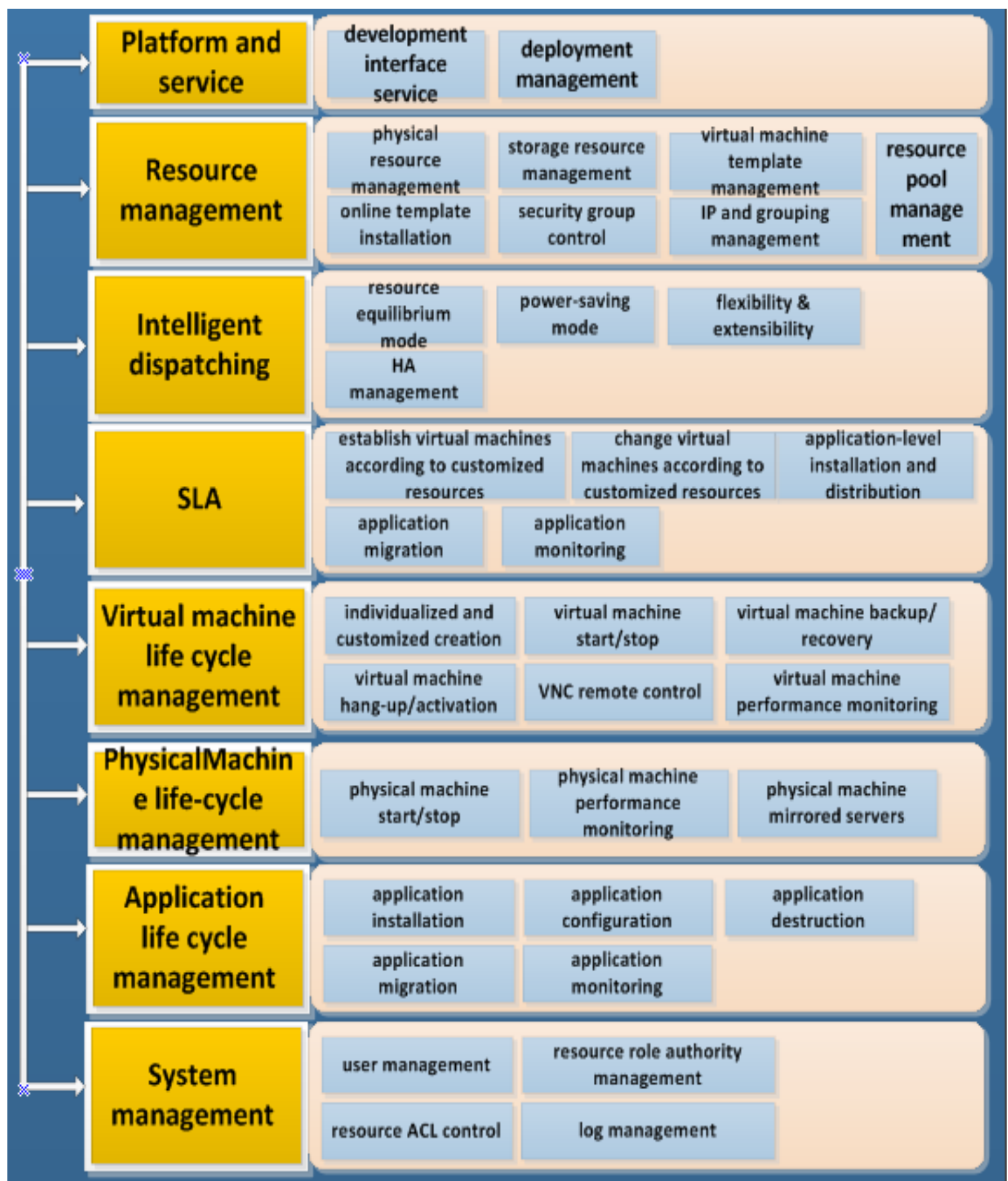

Figure 1:Application Infrastructure

The hardware resource pool system based on domestic virtualization software is showed in the above figure. The main functions are as follows:

(1) Apply and approve the authorization module based on virtual resource; the system administrator can authorize or terminate the authorization operation concerning the virtual resource application form and change order etc submitted by cloud users. The system will record the related operation log.

(2) The virtual machine life cycle management is the core module of the system. This module is mainly used as virtual resource query, basic operation, backup and recovery, remote control, performance monitoring and dispatching configuration etc;

(3) The physical life cycle management is mainly used to manage the physical machines;

(4) The system management module refers to self-management;

(5) The intelligent dispatching includes the realization of resource equilibrium, power saving mode as well as flexibility \& extensibility; 
The function components can be divided into display interface component (take charge of external integration and application), business function component (take charge of related virtualization operation) and common service component (take charge of system self-management).

section headings are in boldface capital and lowercase letters. Second level headings are typed as part of the succeeding paragraph (like the subsection heading of this paragraph).

\section{CONCLUSION}

Anhui Electric Power Corporation has achieved hardware infrastructure resource co-sharing \& utilization, allocate on-demand, integrated management and dynamic dispatching capability, given full play to equipment efficiency and reduced energy consumption \& operation cost in the domestic virtualization software resource pool construction and application process. At present, Anhui Electric Power Corporation has constructed domestic virtualization software resource pool (grade-2 classified protection, grade-3 classified protection). Further, the company has totally 84 pooling physical host, 137 virtual servers and $22 \mathrm{~T}$ mounts and storage.

The company has formed domestic controlled server virtualization software and established information system hardware resource pool as well as its management system of SGCC with core competitive technical capability. Based on security controllability and cost saving, the company has formed the capability concerning information resource co-sharing \& application, allocate on-demand, integrated management and dynamic dispatching, given full play to equipment effectiveness, reduced operation cost and energy consumption and realized physical resource virtualization, software resource standardization, resource dispatching automation and resource service integration so as to further improve the intensive construction and management level of hardware infrastructure of data center.

\section{References}

[1] Tian Jianwei, Liu Xiaoxiao and Li xi: Application on VMware Esxi virtulization technique in server resource integration.Hunan Electric Power. Vol. 32-6 (2012), p.5

[2] Kusic,Daral;Kephart: Power and performance management of virtulized computing environments cia look ahead control. Cluster Computing. Vol. 12-1(2009), p.1

[3] WeiShan Zhang,LiCheng Chen,Xin Liu,QingHua Lu,PeiYing Zhang,Su Yang. An OSGi-based flexible and adaptive pervasive cloud infrastructure[J]. Science China Information Sciences . 2014 (3)

[4] Jingjun Zhang,Lei Wang,Hui Li,Guangyuan Liu. Research Java Web framework based on OSGi[J]. Procedia Engineering . 2011

[5] Yao Huachao,Wang ZhenYu.Construction of Virtualized Resource Pool Based on KVM-QEMU with Libvirt[J]. Computer and Modernization.2013 